\title{
Depletion of charge produced during plasma exposure in aluminum oxide by vacuum ultraviolet radiation
}

\author{
C. Cismaru, a) J. L. Shohet, J. L. Lauer, and R. W. Hansen ${ }^{\text {b) }}$ \\ Center for Plasma-Aided Manufacturing and Department of Electrical and Computer Engineering, \\ University of Wisconsin-Madison, Madison, Wisconsin 53706
}

\section{S. Ostapenko}

Center for Microelectronics Research, University of South Florida, Tampa, Florida 33620

(Received 12 June 2000; accepted for publication 9 October 2000)

\begin{abstract}
A temporary increase in the conductivity of aluminum oxide sputter deposited on the surface of aluminum wafers was made by exposure to vacuum ultraviolet (VUV) radiation produced by a synchrotron light source. The oxide was charged, either positively or negatively, by exposure to a nonreactive inductively coupled plasma, under typical plasma processing conditions. We show the dependence of the conductivity on the energy of the incoming radiation, and conclude that only those photons whose energy is above the band gap of the oxide are capable of producing a temporary increase in the conductivity. By exposing localized regions of precharged oxide samples to the vacuum ultraviolet radiation, we produce regions of charge depletion in and around the exposed areas. We conclude that VUV radiation may be used to significantly decrease plasma-induced surface charging of dielectrics. (C) 2000 American Institute of Physics.
\end{abstract}

[S0003-6951(00)01250-X]

During plasma processing, charging of dielectrics plays a leading role within the damage mechanisms of semiconductor devices and plasma-processed materials in general. This damage mechanism is greatly influenced by plasmaemitted $\mathrm{x}$ ray, vacuum ultraviolet (VUV), and ultraviolet radiation. ${ }^{1-3}$ It was determined that most processing plasmas emit radiation in the VUV energy band of 4-30 eV, with most of the radiation above $9 \mathrm{eV}$, the latter of which is approximately the energy band gap of $\mathrm{SiO}_{2},{ }^{4}$ and more than the energy band gap of other dielectrics used in semiconductor device manufacturing (e.g., $6 \mathrm{eV}$ for $\mathrm{Si}_{x} \mathrm{~N}_{y}$ and $8.3 \mathrm{eV}$ for $\mathrm{Al}_{2} \mathrm{O}_{3}$ ). The radiation is absorbed in the exposed oxide layers and it results in the generation of electron-hole pairs. Although it has been established that electron-hole-pair generation in the oxide increases the $\mathrm{SiO}_{2}$ bulk and interface trapped-charge density, which may affect device reliability, ${ }^{5,6}$ we believe plasma VUV irradiation of oxides can have a beneficial effect by inducing a temporarily enhanced oxide conductivity. This can reduce dielectric charging, especially that induced by electron-shading effects ${ }^{7}$ during plasma etching of high aspect-ratio devices, by providing a safe way to discharge these structures and, thus, minimize charging damage. The enhanced conductivity can also have beneficial effects on the etching properties of oxides such as reduction of notching, sidewall bowing, and trenching.

It has been previously shown, by exposure to synchrotron radiation in the earlier energy range, that a temporary increase in oxide surface conductivity was produced while the oxide was exposed to VUV radiation. ${ }^{8}$ The purpose of this work is to show the energy dependence of the surface conductivity in $\mathrm{Al}_{2} \mathrm{O}_{3}$ and that an actual depletion of previ-

${ }^{a)}$ Present address: Conexant Systems, Inc., Newport Beach, CA 92660.

b) Also at: Synchrotron Radiation Center, University of Wisconsin-Madison, Madison, WI 53706. ously stored charge occurs in and around the exposed region.

First, we demonstrate the dependence of the $\mathrm{Al}_{2} \mathrm{O}_{3}$ surface conductivity on the energy of the incoming radiation and we show that the peak conductivity occurs for irradiation with photons with an energy of approximately $18 \mathrm{eV}$. Second, by exposing precharged oxide samples to VUV, we show that the regions exposed to VUV radiation exhibit charge depletion on the surface and down a few hundred angstroms below the surface in and around the exposed areas.

It was desired that the investigation of the VUV-induced conductivity on an oxide-coated wafer, as a function of the energy of the plasma VUV radiation, takes place under exposure of the test structures to processing plasmas, while the VUV-emission intensity and wavelength are measured. However, these measurements are not possible during plasma exposure because the plasma provides an additional current path that interferes with our measurements. To eliminate this, we used monochromatic synchrotron radiation in the same photon energy range to expose the test structures under vacuum. The advantage of using monochromatic synchrotron radiation is the ability to determine the dependence of measured quantities on the wavelength of the incoming radiation.

To achieve our first goal, we exposed oxide-coated wafers to synchrotron radiation at the Synchrotron Radiation Center (SRC), at the University of Wisconsin-Madison. The monochromatic light was supplied by the Aladdin synchrotron storage ring, passing through a VUV monochromator. The electron beam used to generate the synchrotron radiation had a current of up to $250 \mathrm{~mA}$, at an energy of $800 \mathrm{MeV}$. The VUV monochromator that was connected to the beamline is a normal-incidence monochromator, in a SeyaNamioka mount, with an output energy range of 4-30 eV and a bandpass of $3 \AA$. 


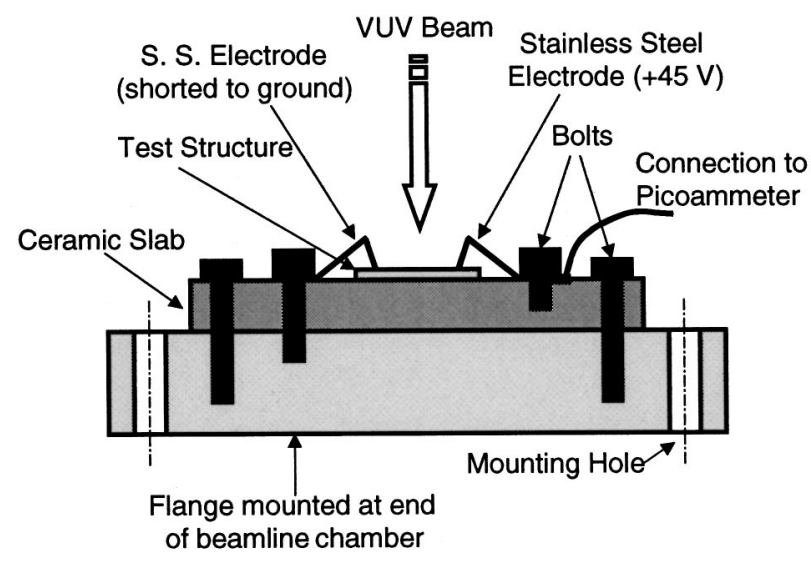

FIG. 1. Experimental setup used for the measurement of oxide surface conductivity under illumination with monochromatic VUV light.

The oxide-coated samples were mounted in a vacuum chamber coupled to the beamline monochromator. The mounting unit was set so as to have normal incidence of the VUV beam on the surface of the wafer. The monochromatic synchrotron light was focused on the sample (the position of the light spot is shown in Fig. 1), with a spot dimension of 5 $\mathrm{mm} \times 15 \mathrm{~mm}$. During the measurements, the chamber was evacuated to pressures in the $10^{-8}$ Torr range. Two stainlesssteel electrodes were used to connect an outside currentvoltage circuit to the sample also as shown in Fig. 1.

The current through the oxide sample was measured at a fixed bias voltage, $45 \mathrm{~V}$, as the synchrotron light energy was varied between 5 and $20 \mathrm{eV}$. The oxide, in this case $\mathrm{Al}_{2} \mathrm{O}_{3}$, has an energy gap of approximately $8.3 \mathrm{eV}$, which is very similar to that of $\mathrm{SiO}_{2}$. The oxide was deposited by reactive sputtering and is $3000 \AA$ thick. The dark current was measured by closing off the light source, but making the same sweep of energy, to make sure that there were no light leaks or other coupling to the circuit. In all cases, the dark current was at least one order of magnitude less than the conduction current. In addition, making sure that the incoming radiation does not hit the electrodes eliminates photoemission currents from being induced in the circuitry. The net oxide current (measured current less dark current) was normalized to a constant electron beam current $(200 \mathrm{~mA})$ and the photon flux at $15 \mathrm{eV}$. The normalized net conduction current as a function of incident photon energy is shown in Fig. 2. Note that no significant increase in conduction current occurs until the VUV photon energy approaches the band gap of the oxide, and we believe that the current due to secondary emission of electrons by the stainless-steel electrodes is small compared to the current produced by electron-hole pair creation in the oxide.

The effect of VUV radiation on depletion of charge is the key part of this work. In order to test this, samples were charged by placing them in a nonreactive argon inductively coupled plasma (ICP) for several minutes. The ICP power was $1 \mathrm{~kW}$ and two different sample bias conditions were used so as to produce a net positive or negative charge in the oxide. The former was achieved with $150 \mathrm{~W}$ radio frequency bias power and the latter with zero bias power. Figure 3(a) shows a scan across the $11.5 \mathrm{~cm}$ sample using the contact potential difference (CPD) technique ${ }^{9}$ that was made before Downloaded 21 Feb 2007 to 128.104.198.71. Redistribution subject to AIP license or copyright, see http://apl.aip.org/apl/copyright.jsp

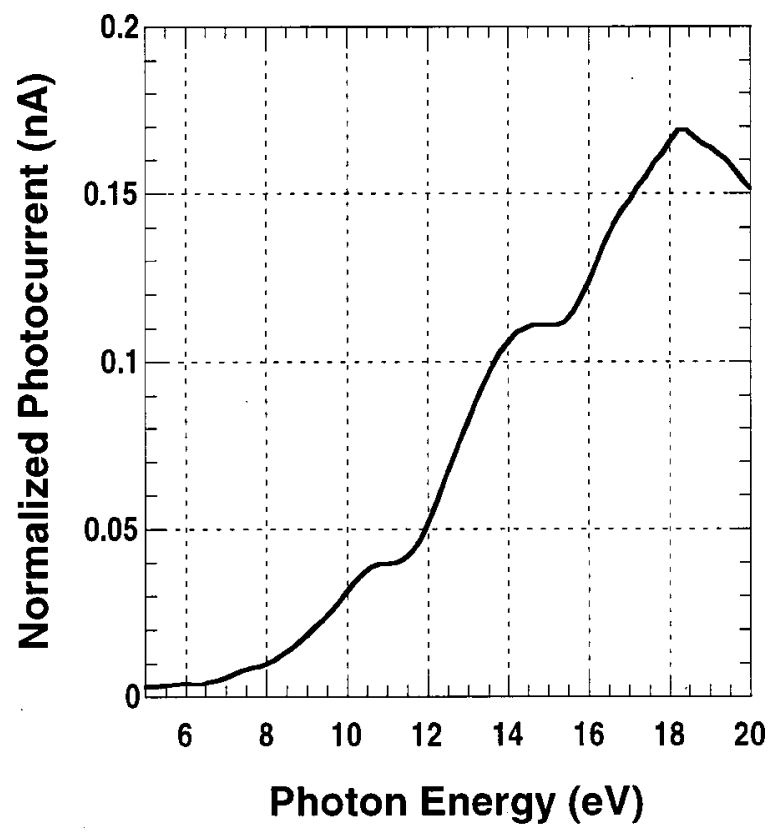

FIG. 2. Photon energy dependence of the normalized net photoconduction current along the $\mathrm{Al}_{2} \mathrm{O}_{3}$ surface.

exposure to the VUV. The difference between positive and negative charging is quite clear.

The CPD technique employed in this work to determine the amount of charging induced by plasma exposure is an application of the Kelvin-probe technique, ${ }^{10}$ used for the measurement of work functions of different materials. The Kelvin-probe method measures the contact potential difference (difference in the work functions) between a vibrating Kelvin probe and the surface under analysis. In our case, by using oxide-coated wafers, the difference between measurements of plasma-exposed samples and nonexposed samples is the change in the oxide surface barrier, a direct function of the charge placed on the oxide's surface by plasma processing. Therefore, plasma induced oxide surface charge density can be expressed as $V_{\mathrm{PDM}} \epsilon_{\mathrm{ox}} / d_{\text {ox }}$, where $V_{\mathrm{PDM}}$ is the CPD potential measurement, $\epsilon_{\mathrm{ox}}$ is the oxide permitivity, and $d_{\mathrm{ox}}$ is the oxide thickness.

Since most plasma processing occurs with a positive charge being placed on the wafer, a CPD scan of a positively charged wafer was made after exposure to VUV, which is shown in Fig. 3(b). Three VUV exposures were made on the sample at $15 \mathrm{eV}$ for 5, 30, and $300 \mathrm{~s}$. The first exposure was made at the exact center of the sample, while the remaining two were displaced above and below the center by about 10 $\mathrm{mm}$. Two CPD scans, at right angles to each other, show a dramatic change in the charge profiles. First, note that there is a significant decrease in charge at the center of the sample and an increase in charge towards the outside of the wafer. We believe that this is as a result of the VUV exposure that produces a temporary conductivity increase both on the surface of the oxide ${ }^{8}$ and in the bulk. ${ }^{11}$ The temporary increase in the conductivity in this area will tend to make this region an equipotential and charge flows outward from the central region to the outside, pushing the other charge ahead of it and resulting in a pileup at the edge of the wafer. In order to achieve this condition, the electron-hole pairs produced by the VUV would have to be sufficiently dense so that ambi- 

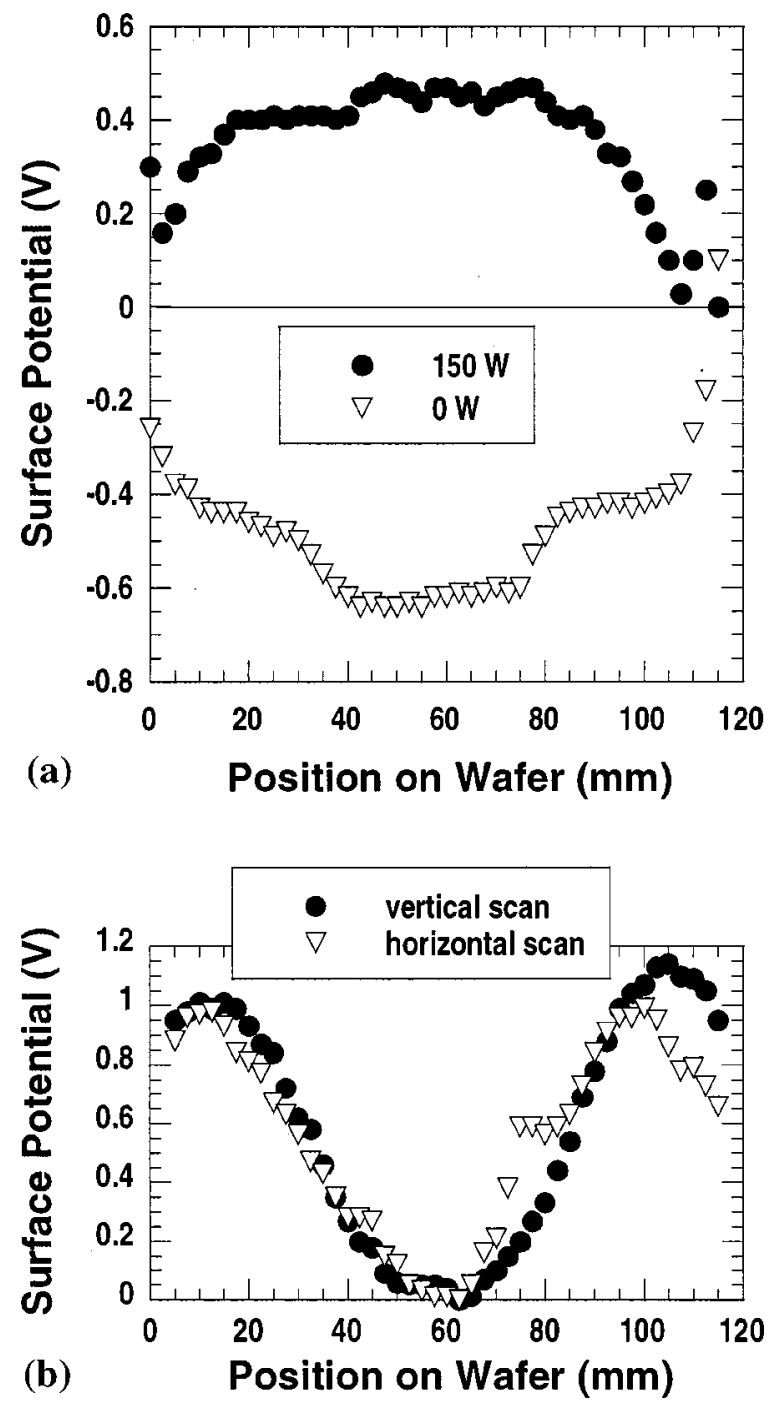

FIG. 3. (a) CPD scans of the plasma exposed samples show positive charge deposited for $150 \mathrm{~W}$ bias conditions and negative charge for no bias conditions; and (b) horizontal and vertical CPD scans of the VUV exposed sample.

polar conditions exist, and thus the long VUV exposures described earlier were made. An explanation of the conductivity mechanism was discussed previously. ${ }^{11}$

When the VUV exposure is ended, the conductivity returns to its pre-exposure state, thus trapping the charge as is shown in Fig. 3(b). We believe that the symmetry in the scans is due to the fact that the first exposure was in the center of the wafer and the majority of the charge movement occurs during the first exposure. In addition, the other exposures were made only $10 \mathrm{~mm}$ above and below the center due to mechanical constraints, a limited distance compared to the dimensions of the wafer. Subsequent exposures, although they can result in a temporary conductivity increase, are incident in a charge-depleted region, and thus do not significantly change the charge distribution. It is reasonable to conclude that, if a conducting path were placed on the edge of the VUV exposed region to ground, the charge would drain off as long as the VUV was turned on, thus providing a means to drain off the accumulated charge.

In summary, we showed the dependence of the $\mathrm{Al}_{2} \mathrm{O}_{3}$ surface conductivity on the energy of the incoming radiation. We found the peak surface conductivity to occur for irradiation with photons with an energy of approximately $18 \mathrm{eV}$. Second, by exposing precharged oxide samples to VUV, we showed that the regions exposed to VUV radiation exhibit charge depletion on the surface and down a few hundred angstroms below the surface in and around the exposed areas. The oxide was precharged, either positively or negatively, by exposure to nonreactive inductively coupled plasma, under typical plasma processing conditions. We conclude that VUV radiation can significantly decrease plasmainduced surface charging of dielectrics.

This work was also supported in part by the National Science Foundation under Grant No. EEC-8721545 and in part by Texas Instruments. SRC is a national facility, funded by the National Science Foundation under Award No. DMR9531009.

${ }^{1}$ R. A. Gdula, IEEE Trans. Electron Devices 26, 644 (1979).

${ }^{2}$ T. Yunogami, T. Mizutani, K. Suzuki, and S. Nishimatsu, Jpn. J. Appl. Phys., Part 1 28, 2172 (1989).

${ }^{3}$ T. Mizutani, in International Symposium on Plasma Process-Induced Damage, edited by K. P. Cheung, M. Nakamura, and C. T. Gabriel (NCCAVS, Sunnyvale, CA, 1996), p. 157.

${ }^{4}$ C. Cismaru and J. L. Shohet, Appl. Phys. Lett. 74, 2599 (1999).

${ }^{5}$ S. A. Bell and D. W. Hess, J. Electrochem. Soc. 139, 2904 (1992).

${ }^{6}$ A. W. Flounders, S. A. Bell, and D. W. Hess, J. Electrochem. Soc. 140, 1414 (1993)

${ }^{7}$ K. Hashimoto, Jpn. J. Appl. Phys., Part 1 32, 6109 (1993).

${ }^{8}$ C. Cismaru, J. L. Shohet, and J. P. McVittie, Appl. Phys. Lett. 76, 2191 (2000).

${ }^{9}$ P. Edelman, A. M. Hoff, L. Jastrzebski, and J. Lagowski, Proc. SPIE 2337, 154 (1994).

${ }^{10}$ W. A. Zisman, Rev. Sci. Instrum. 3, 367 (1932).

${ }^{11}$ C. Cismaru and J. L. Shohet, J. Appl. Phys. 88, 1742 (2000) 